\title{
Rewiring the home
}

\author{
Following the successful deployment of photonics in long-haul communications, the \\ technology is now making its way down the food chain and changing the way data is brought \\ into the home, and perhaps ultimately passed around it.
}

Flick through the pages of this month's issue and it will become immediately apparent that a strong theme throughout is optical communications. Arguably one of the most important applications of photonics technology, it has been responsible for helping to drive the development of low-loss optical fibre, high-performance laser diodes, fast modulators and integrated optics, to name just a few examples. In fact, it is almost certain that photonics today would not be nearly as advanced as it is without this driver.

Inside this issue we bring together a diverse mixture of articles covering various aspects of optical communications everything from the latest breakthroughs in devices through to discussion of important emerging technologies and applications.

In the main journal, we have a peerreviewed primary research paper on a high-performance hybrid optical modulator that is made by bringing together organic and inorganic materials ${ }^{1}$. Our review article takes a look at the latest developments in quantum communication, where single photons are used for transferring information and data processing ${ }^{2}$.

We also have a commentary describing how optical networking today is allowing scientists around the world to interact by sharing huge amounts of data in real time ${ }^{3}$. This is opening the door to high-definition video conferencing, analysis of results from data-rich experiments in particle physics or astronomy, as well as telemedicine and grid computing.

Our Technology Focus is dedicated to the topic and describes the progress that has been made in fibre-to-the-home (FTTH) technology, a view of how optical networks may look in 2015 and the prospects for short distance optical interconnects ${ }^{4}$. This is combined with a

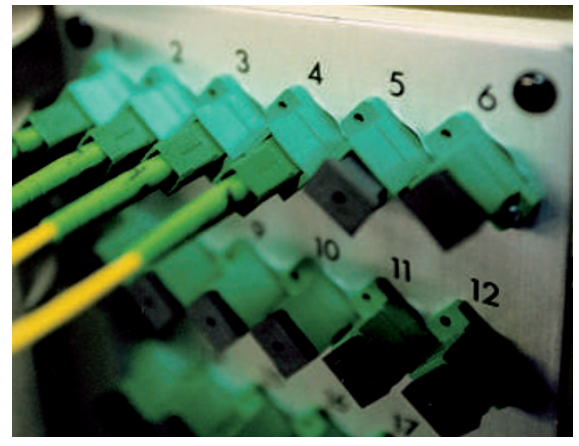

round-up of the latest business and product news from the sector.

Despite suffering a market crash at the turn of the millennium, which resulted in the closure of many optical start-ups and well-established firms, the opticalcommunications sector now seems to be back on track, albeit in a slightly different direction. Although much of the initial investment in optical communications was focused on developing technology for long-haul transmission between cities and across oceans, the most exciting prospect right now is moving the technology closer to the home and ultimately electronic and computer interfaces.

The reality is that our lust for highquality digital information (high-definitiontelevision streams, large megapixel camera images, vast collections of MP3 music files) will soon be causing problems for copper wires that are getting close to their maximum achievable bandwidths. In short, electrical connections that bring data into the house and transfer it between electronic and computer equipment are struggling to keep up ${ }^{5}$ and the ultimate answer lies with optical alternatives that have the potential to scale to much faster data-transfer speeds.

Let's take a look at some numbers. Telephone-wire-based broadband technologies to the home will probably be limited to around 10-100 $\mathrm{Mbit} \mathrm{s}^{-1}$ (ref. 5) depending on the reach from the local exchange, and today, many people are limited to just a few megabits per second. At the same time, transmission over copper connections between electronics starts to get very difficult for speeds faster than around $20 \mathrm{Gbit} \mathrm{s}^{-1}$ (ref. 6). In contrast, FTTH technologies can already support bandwidths of $100 \mathrm{Mbit} \mathrm{s}^{-1}$ to $1 \mathrm{Gbit} \mathrm{s}^{-1}$ to the premise. In addition, experiments suggest that parallel optical interconnects could open the door to data-transfer rates of hundreds of gigabits per second for short links between electronic equipment by using arrays of laser transmitters.

In fact it is more than probable that future versions of popular data interfaces to succeed USB (universal serial bus), Firewire (IEEE 1394) and HDMI (high-definition multimedia interface) will at some point need to become optical to reach the required data-transmission rates. The real challenge for optical technologies is not scaling to sufficient speeds, but rather having a design that is cost-effective, mass-producible, robust and practical. The question is not if photonics will rewire the home and electronics, but when will the switch take place. That answer will be dictated by economics, just as it was for long-haul communications. The fact that digital audio has already started to make the transition and that one of the most common interfaces is based on a plug-in polymer optical-fibre design is very promising.

\footnotetext{
References

1. Enami, Y. et al. Nature Photon. 1, 180-185 (2007).

2. Gisin, N. \& Thew, R. Nature Photon. 1, 165-171 (2007).

3. Smarr, L. Nature Photon. 1, 133-135 (2007).

4. Nature Photon. 1, 147 (2007).

5. Twist, K. Nature Photon. 1, 149-150 (2007).

6. Alduino, A. \& Paniccia, M. Nature Photon. 1, 153-155 (2007).
} 